\title{
AV-Medien in der religiösen Bildungsarbeit: Funktionen und Kriterien für Auswahl und Einsatz
}

\author{
von Anton Täubl
}

\section{Wachsende Bedeutung der $A V M$ in religiösen Lernprozessen}

Die Bedeutung von audiovisuellen Medien (AVM) für religiöse Lernprozesse und für kirchliche Bildungsveranstaltungen ist in den letzten Jahren deutlicher hervorgetreten. Immer mehr Religionslehrer und Seelsorger, Erzieher und Bildungsreferenten im kirchlichen Dienst verwenden Schallplatten, Tonbilder, Kurzfilme und andere audiovisuelle Materialien in ihrer täglichen Arbeit. Es gibt zahlreiche Gründe für diese Entwicklung; sie können hier nicht alle aufgeführt werden. Auf drei Gesichtspunkte möchte ich aber doch aufmerksam machen:

Der erste Grund für die zunehmende Verwendung audiovisueller Medien ergibt sich aus der Veränderung der gesellschaftlichen Kommunikation. Mit der Einführung des Fernsehens - in der Bundesrepublik Deutschland im Herbst 1953 - wurde der Prozeß einer weitgehenden Visualisierung der Informationsvermittlung eingeleitet. Bei der Übermittlung von Informationen ist eine Verlagerung vom Abstrakten zum Konkreten, vom Begriff auf den Gegenstand selbst, vom Text auf das Bild, eingetreten. Das Sichtbare, Anschauliche, Bildhafte bekommt allmählich das gleiche Gewicht wie der verbale Vortrag oder das gedruckte Wort. Versteht man nun Lehren und Lernen im weitesten Sinn als Verbreitung und Aufnahme von Informationen, so ergibt sich, daß auch die religiöse Bildungsarbeit der gesamtgesellschaftlichen Veränderung im Informationsbereich Rechnung tragen muß.

Der zweite Grund für die verstärkte Verwendung von Medien hat seine Wurzeln im Bereich der Pädagogik - vor allem der Lernpsychologie. Die intensivere Beschäftigung mit den Voraussetzungen und Bedingungen des Lehrens und Lernens hat dazu geführt, daß die psychologischen und soziologischen Komponenten der Kinder in ihrer Bedeutung für Lernbereitschaft und Lernerfolg klarer erkannt wurden; in dem Zusammenhang wurden dann auch kooperative Unterrichtsformen und audiovisuelle Medien aufgewertet, weil sie die Lernenden ganzheitlicher motivieren und intensiver am Lernprozeß beteiligen.

Ein dritter Grund für die wachsende Bedeutung audiovisueller Medien ergibt sich aus der Reflexion über die Vermittlungsprozesse in der Verkündigung. Es geht heute $\mathrm{ja}$ in der Tat nicht nur um die verständliche Formulierung theologischer Aussagen; vielmehr müssen sich die Theologen auch moderner, der heutigen Situation angemessener Methoden bei der Vermittlung der christlichen Botschaft bedienen. Audiovisuelle Medien und massenkommunikative Vermittlungssysteme bieten hier Chancen an.

Dipl. Päd. Theol. Anton Täubl ist im Referat AV-Medien in der Zentralstelle für Medien in Bonn tätig und Leiter des Referates "Katholische Religion" beim Institut für Film und Bild in Wissenschaft und Unterricht in München/Grünwald. 


\section{Allgemeine Funktionen audiovisueller Medien in Lernprozessen und ibre Grenzen}

Bildungsarbeit als Lernprozeß

Bildungsarbeit - und damit auch Unterricht in der Schule - ist ein absichtsvolles Lehr- und Lerngeschehen. Im Unterschied zum Alltagslernen, wo zufällige Erlebnisse vorwiegend unplanmäßig verarbeitet werden, wird in der Bildungsarbeit die Begegnung der Lernenden mit dem Unterrichtsgegenstand planmäßig herbeigeführt. Im Unterricht aber ist die Wirklichkeit, mit der man die Schüler konfrontieren will, zumeist nicht präsent. Der Augenblick, in dem sie sich zufällig einstellt, kann nicht abgewartet werden. Deshalb müssen verfügbare Mittel zur Repräsentation der Wirklichkeit im Unterricht bereitgestellt werden. Jede Bildungsarbeit ist demnach auf Mittel, d. h. auf Medien zur Vergegenwärtigung des Lerngegenstandes angewiesen. Dies um so mehr, als heute an die Stelle von Primärerfahrungen - also an die Stelle der Begegnung mit dem unmittelbaren Leben - sekundäre Erlebnisse treten: Bücher, Fotos, Filme, Experimente, Modelle usw. ersetzen die Konfrontation der Lernenden mit der ursprünglichen Realität. Das aber bedeutet, daß Unterricht und Bildung ohne sekundäre Systeme nicht mehr möglich sind. Lehr- und Lernmittelsammlungen - und dazu gehören in zunehmendem Ausmaß audiovisuelle Medien - werden zum entscheidenden Umschlageplatz für jede effektive Bildungsarbeit.

Möglichkeiten und Grenzen audiovisueller Medien

Das gegenwärtige Medienangebot für Schule und außerschulische Bildungsarbeit reicht von der Folie zum Tageslichtprojektor bis zum computerunterstützten Lehrsystem. Es liegt auf der Hand, daß die verschiedenen Medien jeweils spezifische Möglichkeiten und Grenzen, Probleme und Schwierigkeiten mit sich bringen. Zum Teil erfordert das unterschiedliche Maßnahmen, wenn ihr optimaler Einsatz angestrebt werden soll. Neben spezifischen Aspekten gibt es eine Reihe von vergleichbaren Gesichtspunkten. Nach den bisherigen Erfahrungen bieten audiovisuelle Medien im Unterricht folgende Möglichkeiten:

a) AV-Medien können Lehrfunktionen übernehmen;

b) durch AV-Medien lassen sich bestimmte Lehrfunktionen im Rahmen des Bildungsprozesses optimieren;

c) AV-Medien können im Rahmen geeigneter Unterrichtsformen und im Verbund - z. B. als Medienpakete - wirkungsvolleren Unterricht leisten als der Lehrer allein;

d) AV-Medien können zu einer Rhythmisierung der Lehr- und Lernprozesse in angespannte und entspannte, in aktive und rezeptive, in individuelle und soziale Phasen beitragen;

e) AV-Medien können unter bestimmten Voraussetzungen die Motivation der Lernenden fördern;

f) AV-Medien erleichtern die Differenzierung des Lernprozesses und die Individualisierung der Lernprozesse, z. B. im programmierten Unterricht;

g) AV-Medien stellen in bezug auf bestimmte Lehrfunktionen eine optimale Unterrichtsvorbereitung dar, man denke z. B. nur an die Zusammenarbeit von Fachleuten bei der Erstellung, Erprobung und Revision von Produktionen;

h) AV-Medien als objektivierte Lehrfunktionen können beliebig vervielfacht werden (Multiplikationseffekt). 
Diese Möglichkeiten finden ihre Grenzen zum einen in den jeweiligen organisatorischen, personellen und finanziellen Voraussetzungen, denen Bildungsprozesse unterworfen sind; sie ergeben sich zum anderen aber auch aus pädagogischen und didaktischen Erwägungen. Die Grenzen und möglichen Nachteile des Medieneinsatzes führen zu den offenen Problemen der heutigen Praxis. Hier möchte ich zunächst nur auf drei grundlegende Gesichtspunkte aufmerksam machen:

a) Durch Einzelmedien lassen sich immer nur ganz bestimmte (medienspezifische) Lehrfunktionen optimieren und nicht der Lehrprozeß als ganzer. Die AV-Medien müssen sich demnach untereinander und mit anderen Unterrichtsformen ergänzen.

b) Der Lehr- und Lernprozeß ist wesentlich auf personale Interaktions- und Kommunikationsformen angewiesen. Medieneinsatz - als nichtpersonale Kommunikationsform - kann jene nicht ersetzen und muß deshalb in personale Unterrichtsformen eingebettet werden (vgl. Bedeutung des Gesprächs beim Medieneinsatz).

c) Der Einsatz vorgefertigter Medien setzt in der Regel die Ubernahme von Entscheidungen Dritter über einzelne Lehrziele, Inhalte und Methoden voraus. Diese können den Zielen und Interessen der Lehrer und Schüler in der konkreten Klassensituation zuwiderlaufen. Das macht vermittelnde Prozesse zwischen Lehrzielen der Hersteller von AV-Medien und Unterrichtszielen der jeweiligen Gruppe oder Klasse notwendig.

\section{Die spezifische Funktion audiovisueller Medien im religiösen Lernprozeß}

Religion und Theologie sind meiner Ansicht nach nicht als ein Bereich neben anderen Wirklichkeitsbereichen anzusehen. Sie sind vielmehr eine Dimension unseres Lebens, unter der Wirklichkeit in all ihren Erscheinungsformen erfaßt und befragt werden kann. Von daher läßt sich als Kriterium für Auswahl und Einsatz von audiovisuellen Medien in der religiösen Bildungsarbeit der Satz formulieren: Alle AVM, in denen es um den Menschen geht - wie z. B. um Fragen nach seiner Herkunft und Bestimmung, seinen Grenzen und Ängsten, seinen Freuden und Hoffnungen, den Normen für sein Verhalten und seinen religiösen Außerungen usw. - , sind für den Bereich theologischer Reflexion und Auseinandersetzung von Bedeutung. Im einzelnen sind demnach Medien für den Religionsunterricht relevant

- wenn sie die Begegnung mit dem Unterrichtsgegenstand möglichst interessant, anschaulich und sachadäquat herbeiführen;

- wenn sie die Schüler für menschliche Grunderfahrungen sensibilisieren;

- wenn sie die religiösen Dimensionen des Lebens ansprechen oder aufdecken;

- wenn sie verschiedene Deutungs- und Lösungsmöglichkeiten im Blick auf die Bewältigung von Wirklichkeit anbieten bzw. zur Diskussion stellen;

- wenn sie Fragen auslösen, die für das Selbstverständnis des christlichen Glaubens Bedeutung haben;

- wenn sie die Erlebnisfähigkeir fördern und unter Umständen auch Betroffenheit auslösen;

- wenn sie die Erhellung der emotionalen Befindlichkeit ermöglichen und die Schüler dazu ermuntern und ihnen helfen, eigene Empfindungen, Gedanken, Erinnerungen usw. unbefangen auszusprechen;

- wenn sie Impulse für ein verantwortliches Handeln geben;

- wenn sie die religiöse Sprach- und Ausdrucksfähigkeit fördern; 
- wenn sie biblische Inhalte oder christliches Brauchtum auf neue Weise - oder überhaupt - zur Diskussion stellen;

- wenn sie dogmatische Aussagen in einer neuen Perspektive zeigen (Verfremdungseffekt);

- wenn sie eine meditative Grundhaltung begünstigen;

- wenn sie Verständnis für andere religiöse und weltanschauliche Lebensformen wecken und dadurch zu einer kritischen Toleranz beitragen.

Audiovisuelle Medien müssen sich also nicht von vorneherein und in jedem Fall durch spezifisch christliche Gehalte als sogenannte „religiöse Produktionen“ ausweisen. „Das Christliche" bzw. „das Religiöse" läßt sich eben nicht als ein Bereich der Wirklichkeit sehen, der sich gegen andere Bereiche abgrenzen und einseitig isoliert darstellen läßt.

Aus dem eben Gesagten ergibt sich aber auch, daß der Umgang mit AV-Medien theologisch nicht verantwortet werden kann,

- wenn der Lerninhalt anthropologisch und theologisch nicht sachgemäß dargestellt ist;

- wenn den Schülern die Einsicht in Zusammenhänge erschwert wird (Ubereinstimmung der Struktur eines Mediums mit dem Lerngegenstand);

- wenn einseitig beeinflussende Medien nicht durch geeignete Kontrastmedien ergänzt werden, so daß den Schülern die Möglichkeit der kontroversen Auseinandersetzung und der freien Entscheidung eröffnet wird (Fragen der Manipulation);

- wenn angstfreie Kommunikation zwischen Schülern und Lehrern und den Schülern untereinander nicht gewährleistet ist;

- wenn durch irgendwelche Umstände die Ehrlichkeit im Umgang mit den eigenen Gedanken beeinträchtigt wird;

- wenn Sachverhalte, die keine allgemein gültige und absolute Wertung zulassen, vom Lehrer abschließend bzw. als nicht mehr hinterfragbar beantwortet werden;

- wenn die Schüler zur Annahme bestimmter Formen und Werte (Moralvorstellungen, Glaubensinhalte) kritiklos verleitet oder gar gezwungen werden;

- wenn der Lehrende es bewußt vermeidet, seine eigene Ubberzeugung mitzuteilen und sie gleichzeitig in Frage stellen zu lassen.

\section{Beispiele}

\section{Der Realitätsbezug}

Eine wichtige Aufgabe der Schule ist darin zu sehen, den heranwachsenden Menschen zu einem aufgeklärten Weltverständnis zu führen. In der Bildungsarbeit geht es demnach um Erschließung und Interpretation der Wirklichkeit, um einen Beitrag zur kritischen Bestimmung des eigenen Standpunktes innerhalb der Gesellschaft. Dies setzt eine intensive und vieldimensionale Analyse der subjektiven und objektiven Welt des jungen Menschen voraus. Die Lebenswirklichkeit soll im Religionsunterricht in ihrer Tiefendimension, in allem was ihr Sinn und Bestand gibt, zur Sprache kommen. Das Leben, wie es den Menschen trifft und betrifft, das ihn vor die Sinnfrage stellt und ihm Antwort abfordert und Verantwortung auferlegt, ist der Pol religiöser Bildungsarbeit.

Nun wird aber gerade dem Religionsunterricht häufig vorgeworfen, daß er diesem Anspruch nicht gerecht werde. Er sei vielmehr lebensfremd und wirklichkeitsfern. 
Ihm fehle der Bezug zur Zeit. Er gehe am Daseinshorizont des modernen Menschen vorbei. Die Ausrichtung der christlichen Botschaft vollziehe sich nicht oder viel zu wenig als Interpretation des gelebten und erlebten Lebens. Ja die Theologie erliege immer wieder der Versuchung, sich eine Sonderwelt in einer "splendid isolation ${ }^{\text {" }} \mathrm{zu}$ konstruieren, die dem Menschen der Industrie- und Konsumgesellschaft als Fremdkörper erscheint.

Diese Argumentation - besteht sie nun zu recht oder zu unrecht - ist beachtlich, Die Frage für uns heißt: Können AVM hier Abhilfe schaffen?

Zweifellos eignet den audiovisuellen Medien ein Realitätseffekt. Das gilt insbesondere für Film und Fernsehen. Dieser Realitätseffekt besteht darin, daß wir das in Laufbildern Gebotene als Wirklichkeit - und dazu als im Augenblick Geschehendes - erleben. Wir werden bis zu einem gewissen Grad am Ereignis selbst beteiligt, angesprochen, zur Stellungnahme herausgefordert. AVM vermitteln eindrucksvoll durch ihre anschauliche, dramaturgisch erlebnishafte Gestaltung bestimmte Lebenssituationen als gemeinsame Ausgangsbasis für das Unterrichtsgeschehen. Sie aktualisieren menschliche Grunderfahrungen und rühren dadurch die Rezipienten an. Auf diese Weise wird die Nur-Unterweisung im Religionsunterricht überwunden.

Die Bildmedien bedienen sich der Fotografie. Diese ist durch einen extremen Konkretheitsbezug ausgezeichnet. Das Live-Foto wirkt als Abbild der Wirklichkeit. Durch das Dokumentarfoto übernehmen Fakten das Gewicht der Begriffe. So gibt die Audiovision dem Menschen den Blick frei für die Realität des Seins und zwingt ihn zur Begegnung mit dem Alltag. Sie konfrontiert ihn nicht mit abstrakten, blutleeren Ideen, Theorien, Ideologien, verbalen Abhandlungen, sondern mit einem konkreten Stück unserer Erde.

Der Realitätsbezug der AVM trägt sicher zu einem lebensnahen Religionsunterricht bei und führt dadurch zu seiner Aufwertung innerhalb der Schule.

Förderung der religiösen Sprach- und Ausdrucksmöglichkeiten

Die traditionellen Vollzugsformen religiöser Erziehung - Gebet in der Familie, Gottesdienstbesuch, Lektüre der Bibel, christliches Brauchtum usw. - sind so sehr zurückgegangen, daß vielfach kaum noch nennenswerte Restbestände übrig geblieben sind. Wo Erwachsene es wagen, außerhalb des Religionsunterrichts in Gesprächen mit Kindern und Jugendlichen das übliche religiöse Vokabular zu gebrauchen, wirkt sich das geradezu hemmend, oft sogar lächerlich aus. Das religiöse Vokabular hat seine Durchschlagskraft verloren. Der innerkirchliche Jargon ist abgegriffen, mit falschen Vorstellungen belastet. Die theologische Fachterminologie von Gnade, Himmel, Hölle, Sünde, Erlösung und Nächstenliebe besagt dem modernen Menschen nicht mehr viel und wird weithin überhaupt nicht mehr verstanden. Die Sache aber, um die es hier geht, ist für den Menschen nach wie vor höchst bedeutsam. Wir müssen deshalb nach Möglichkeiten suchen, um diese Lebensbereiche sprachlich neu einzuführen. Wir benötigen neue Ausdrucksformen, um das Evangelium verständlich zu machen.

Hier bietet sich die Audiovision als neue Sprache des Glaubens an. Die Hauptfunktion von Bild und Klang besteht nicht in Definitionen, nicht einmal in der Illustration, sondern darin, daß bestimmte Zusammenhänge angedeutet werden. Das Bild deckt durch Assoziationen, die im Bereich des Verstandes und der Gefühle wachgerufen werden, Sinnzusammenhänge auf und stellt eine Verbindung zur konkreten Umwelt her. Sittlich relevante Fragen und religiöse Dimensionen werden durch das 
vorhandene Medienmaterial angedeutet, indem Gegebenheiten aus dem alltäglichen Leben aktualisiert werden. Solche Grunderfahrungen werden im Unterrichtsverlauf weiter differenziert und besprochen. Erst nach dieser Vorfeldarbeit werden dann theologische Begriffe, die sich auf solche Erfahrungen beziehen, wieder neu verstanden.

Sehr schön läßt sich dieser Vorgang anhand des Kurzfilms "Ein Platz an der Sonne“ aufzeigen: $Z$ wei Männchen treffen sich an einem sonnigen Plätzchen, um das sie sich bald heftig streiten. Dabei verdunkelt sich die Sonne. Die Männchen frieren und sehen erst dann ein, daß sie etwas falsch gemacht haben. Sie bereuen ihre Schuld und die Sonne dringt wieder durch. Die beiden Kontrahenten versöhnen sich und sind glücklich. - Dieser Kurzfilm wird häufig auch zur Bußerziehung für Kinder in der Grundschule eingesetzt. Zentrale Begriffe wie Streit bzw. Sünde, Folgen des Streits, Reue, Wiedergutmachung, Vergebung und Versöhnung können anschaulich verdeutlicht werden. Die Kinder arbeiten dabei selbst die entscheidenden Abschnitte heraus und kommen von sich aus auf die zentralen Begriffe.

Es kommt hinzu, daß neben der empirisch-beschreibbaren Sprache andere Sprachspiele an Bedeutung gewinnen. Das gilt vor allem für die Symbolsprache. „Die Symbolsprache hat im menschlichen Gespräch einen dauernden Platz, weil sie in die Tiefe des menschlichen Erlebens hinabreicht und die Wahrnehmungen und Empfindungen des Menschen über die ganze Daseinsordnung, in der er sich befindet, auszudrücken vermag. ${ }^{\text {} 1}$ Ausgewählte Fotos und Einzeldias, aber auch zahlreiche Film- und Fernsehproduktionen vermögen durch ihre symbolhafte Darstellungsweise die Wirklichkeit in ihrer Transparenz zu zeigen. Aufgabe des Religionsunterrichts wird es sein, Menschen zu jener Transzendenz hinzuführen, die das Vorhandene übersteigt und zur geistigen Wirklichkeit vordringt. Gerade im Gespräch kann durch die Symbolanalyse geistige Wirklichkeit meditiert werden. Immer wieder kann man feststellen, wie sehr das Bildsymbol dem Menschen der Gegenwart entspricht. Natürlich macht die Symbolsprache die empirisch-beschreibbare nicht überflüssig. So wenig wie die Symbolsprache die notwendige realistische Funktion leisten kann, so wenig vermag die realistische Sprache die notwendige Symbolfunktion zu vollziehen.

Es geht auch hier nicht um ein Entweder/Oder, sondern um ein Sowohl/Als auch.

\section{Sensibilisierung für menschliche Grunderfahrungen}

Eine Aufgabe religiöser Erziehung besteht in dem Versuch, den jungen Menschen so zu aktivieren, daß er nicht an der Vordergründigkeit der Ereignisse kleben bleibr, sondern auch ihre Hintergründigkeiten wahrnimmt, um seine Erfahrungen in ihrer Transparenz zu erkennen. Es geht im Religionsunterricht vor allem um die Konfrontation mit der Totalität der menschlichen Lebenswirklichkeit, um die Aufdeckung der Fragwürdigkeit der menschlichen Existenz - und nicht um deren Reduktion auf bestimmte Bereiche des Lebens. Das setzt die Bereitschaft voraus, sich überhaupt mit Grundfragen des Lebens zu befassen. Erfahrungen von Geburt, Hoffnung, Liebe, Freundschaft, Angst, Glück, Schuld, Vergebung, Leid, Zufall, Vertrauen, Verantwortung, Sorge, Scheitern, Spiel usw. fordern den Menschen als Wesen an, das Werte sieht, sich an ihnen orientiert und sich an sie bindet. Schüler sollen deshalb in einem Akt der Befreiung menschliche Grundsituationen erfahren und formulieren lernen, die den Antworten des Glaubens vorausgehen. Sie sollen sich der Herausforderung solcher Situationen wissend und wertend, problembewußt und ihrer selbst bewußt stellen. Nur so wird allmählich deutlich, daß es der religiösen Erziehung um einen 
Dienst am konkreten Menschen geht, um Hilfe zu seiner Selbstfindung und Selbstverwirklichung; daß sie dazu beiträgt, daß sein Leben gelingt, indem er zu einer über alles Vordergründige hinausreichenden Sinngebung und Motivation findet. Die anthropologische Orientierung religiöser Erziehung ist nicht nur eine Frage der Optik, sie ergibt sich aus der Sache selbst.

Diesen Anforderungen stehen aber heute, wie jeder weiß, besondere Schwierigkeiten entgegen. Unsere durchrationalisierte und konsumwütige Welt bietet zwar viele Chancen der Entfaltung; zugleich sind aber viele Unwelteinflüsse dazu angetan, eine progressive Verkümmerung des Menschen zu bewirken. Das pragmatische Zweckdenken und die einseitige "Verkopfung“ des Menschen durch den Verlust an Begegnung mit der Natur und mit den Mitmenschen hat von vielen Zeitgenossen Besitz ergriffen und prägt ihr Denken und Verhalten. Da ist nur noch wenig Raum für ursprüngliche Erfahrungen, für Unvorhergesehenes und Hinterfragbares.

Durch den Einsatz audiovisueller Medien kann die Überintellektualisierung unseres Unterrichts abgebaut werden. Eine besondere Rolle kommt in dem Zusammenhang dem Nacherleben von Motivationsprozessen zu. Allen Entscheidungen des Menschen geht ja eine längere Entwicklung in der Meinungs- und Urteilsbildung voraus. Dabei stehen sich häufig Alternativen gegenüber. Das Soll-Erlebnis - in dem sich das Gewissen manifestiert - weist auf eine Möglichkeit als die richtige hin. Mit Hilfe des Hörspiels, eines spannenden Tonbildes, des Spielfilms können die vom Gewissen als richtig erkannten Möglichkeiten entsprechend motiviert und zum Durchbruch gebracht werden. Meist sind sie durch AVM angebotenen Motive um vieles wirksamer als die nur verbal vermittelten.

AVM stellen auch Verhaltensmodelle zur Diskussion. Auf Schallplatten, Tonbändern und in Filmen werden von Figuren Entscheidungen getroffen. Vom Hörer bzw. Zuschauer werden diese nachvollzogen und durch Introjektion eingeseelt. Sie versickern im Unterbewußten und werden wirksam, wenn Schüler in ähnliche Lebenssituationen geraten. So ist es möglich, bereits zukünftige Lebenssituationen vorweg zu nehmen und entsprechende Entscheidungen vorzubereiten.

Vor allem Kurzfilme erweisen sich als Deuter menschlicher Existenz und als Spiegelung der Zeit. Nach einer Untersuchung von Professor Zöchbauer wurde im Jahre 1969 in $58 \%$ aller Filme und im Jahr 1970 in $42 \%$ aller Filme die Frage nach dem Sinn des Lebens gestellt ${ }^{2}$. Jeder Film, der mit einem Stück Leben konfrontiert, der Wirklichkeit erschließen hilft, der den Horizont erweitert und anregt, nach dem Grund des Daseins Ausschau zu halten, ist aktuell und von Bedeutung für die religiöse Bildungsarbeit.

\section{Kriterien zu Auswabl und Einsatz audiovisueller Medien in der religiösen Bil- dungsarbeit}

In der „Berliner Schule“ werden Medien innerhalb des didaktischen Konzeptes als eigenständiges Entscheidungsfeld angesehen. Nach dem Strukturmodell des Lehrens und Lernens von Heimann/Schulz macht jeder Unterricht vier Entscheidungen des Lehrers erforderlich: Für eine Zielsetzung, einen Inhalt, ein Verfahren und ein Medium; diese Entscheidungen sind ihrerseits abhängig zu machen von anthropologisch-psychologischen und sozial-kulturellen Vorgegebenheiten ${ }^{3}$. Soweit ich sehe, werden Medien hier erstmals ihrer Bedeutung entsprechend von der Didaktik gewürdigt. 
Uns interessiert nun die Beziehung der Medien zu den anderen Momenten des Unterrichts (Interdependenz). Es geht um die alltägliche Frage: Worauf hat der Lehrer bei seiner Entscheidung für den Medieneinsatz besonders zu achten? Wir suchen nach Kriterien für die Medienauswahl und Medienintegration in Unterrichtsabläufe.

\section{Orientierung an Global- und Feinzielen}

Es ist inzwischen eine allgemeine Erkenntnis, daß bei der Bestimmung von Unterrichtsaufgaben die Ziele (Intentionen, Leitideen) den Inhalten (Stoffauswahl und Stoffzuordnung) vorausgehen sollen.

Für die Auswahl der AVM hat das zur Folge, daß sie im wesentlichen abhängig sind vom Bildungsbegriff der Veranstalter: Ihr Einsatz erfolgt in Übereinstimmung mit den curricularen Vorentscheidungen, die das gesamte Bildungswesen beeinflussen zumindest dürfen sie diesen Zielen nicht entgegenstehen.

In dem Zusammenhang wird von monovalenten und polyvalenten Medien gesprochen. Monovalent nennt man Medien, die so beschaffen sind, daß sie nur zur Erreichung eines konkreten Lernzieles herangezogen werden können; ihre Aussage ist einlinig, für die Schüler unmißverständlich; diese Medien erfüllen in eindeutiger Weise ihre Funktion als Verständigungsmittel in der Unterrichtssituation. Polyvalente Medien dagegen sind von ihrem Inhalt oder ihrer Aufmachung her vieldeutig; sie sind deshalb auch meist vielseitig einsetzbar; sie tragen wegen ihrer Mehrdimensionalität zur Erreichung unterschiedlicher Lernziele bei - auch solcher Unterrichtsziele, die im konkreten Fall gar nicht beabsichtigt sind.

Einordnung in die jeweilige Phase des Lernprozesses

Bildungsabläufe setzen sich aus verschiedenen Phasen zusammen. AVM können in allen Phasen die Attraktivität und Effektivität von Lernprozessen steigern.

\section{Erhebung des Vorwissens und der Einstellung}

Dem Religionsunterricht wird häufig vorgeworfen, daß er lebensfremd und wirklichkeitsfern sei; daß ihm der Bezug zur Zeit fehle; daß er am Daseinshorizont der Menschen vorbeigehe; daß er Antworten auf Fragen gebe, die gar nicht gestellt würden. Ein effektiver, lebensnaher Religionsunterricht wird versuchen, da anzusetzen, wo sich die Schïler befinden - bei ihren Fragen und Problemen. Dazu verhilft eine Situationserhebung. Die Interessen und Bedürfnisse der Schüler, ihr Informationspegel und Einstellungsniveau $\mathrm{zu}$ dem fraglichen Unterrichtsgegenstand soll festgestellt werden. AVM können hier einen Beitrag leisten. Fotos rufen Assoziationen hervor und reizen zum Vergleich. In einer Gruppencollage kann ein Themenfeld dargestellt und anschließend darüber diskutiert werden. Der Wissensüberhang der Schüler wird durch einen Film, der nur Teilinformationen liefert, bzw. Teilaspekte aufzeigt, getestet. In all diesen Fällen regen Impulsmedien zur Stellungnahme an und verschaffen dadurch Einblick in die Mentalität und den Wissensstand der Schïler.

\section{Die Motivationsphase}

Wir wissen, daß Bildung nicht im motivfreien Raum existiert und auch nicht um ihrer selbst willen geschieht. Bildungs- und Lernprozesse sind dann am wirksamsten, wenn 
sie gefühlsmäßig motiviert und vom ganzheitlichen Interesse der Rezipienten getragen werden. In der Eingangsphase von Lernprozessen geht es um Einstimmung, um Hinführung an ein Problem, um die Konfrontation mit der Unterrichtswirklichkeit. Das Problembewußtsein soll geweckt und geschärft, ein Denk- und Lernprozeß eingeleitet werden.

Das Bild - vor allem das Laufbild - motiviert in seiner spannenden und realistischen Darbietung das Lernen weitaus mehr als der Lehrervortrag anhand des Buches oder der Tafel. In dieser Phase eignen sich vor allem Anspiele, Impulsmedien, Fragezeichenfilme usw. zum Einsatz.

\section{Die Phase der Darbietung, Differenzierung und Erarbeitung}

Es handelt sich hier nun um die Entfaltung, Durchdringung und Erfassung, sowie um die kritische Aufarbeitung des Problems. Begriffe, Tatbestände und Vorgänge werden geklärt und Grunderkenntnisse gewonnen. Die aufgeworfenen Fragen werden vom menschlich sozialen, vom weltanschaulich religiösen oder vom christlichen Standpunkt aus durchleuchtet. Dieser Phase des Lernprozesses entsprechen vor allem Informations- und Dokumentationsmedien.

\section{Die Phase der Problemlösung bzw. Transferphase}

Diese Phase setzt sich meist außerhalb des Unterrichts fort. Die Leistung ist meist nicht meßbar und für die Effektivitätskontrolle auch nicht greifbar. Ubung, Anwendung, Verwertung, Transfer des Gelernten in die Lebenswirklichkeit ist hier von Bedeutung. In diesem Abschnitt sollte vor allem die Eigenaktivität der Rezipienten berücksichtigt werden; das kann durchaus dazu führen, daß vom Medieneinsatz überhaupt Abstand genommen wird.

Dennoch können Medien auch hier Hilfe leisten, um eine neue Haltung zu bestärken oder eine Änderung bisheriger Einstellungen herbeizuführen. Gerade Filmsituationen regen die Auseinandersetzung mit dem dargestellten sittlichen Verhalten an. Dadurch können sich Entscheidungen anbahnen. Medien haben hier eine Impulswirkung, einen Aufforderungscharakter zum Handeln. Wichtig in der Transferphase bleibt, daß ein Medium so gestaltet ist, daß seine Lösungsvorschläge auf ähnliche Situationen übertragbar bleiben. Utopien oder wirklichkeitsfremde Darstellungen eignen sich dafür nicht. In der Übertragbarkeit liegt der Wert des Exemplarischen. Transferiert werden können Lösungsmethoden, Arbeitstechniken, Denkschemata, Verhaltensweisen.

Die Phase der Zusammenfassung bzw. der Wiederholung

Lernprozesse ziehen sich oft über viele Stunden bzw. Tage und Wochen hin.

Am Ende eines Unterrichtsverlaufs können audiovisuelle Medien eingesetzt werden, um den Stoff noch einmal zusammenzufassen, um wichtige Aussagen in Erinnerung zu rufen, um das gesamte Lerngeschehen noch einmal zu aktualisieren. Das Tonbild "Martin Luther und die Reformation“ eignet sich beispielsweise recht gut, um im kirchengeschichtlichen Unterricht die Ereignisse der Kirchentrennung noch einmal bewußt zu machen. 


\section{Das Feedback bzw. die Kontrolle der Lernergebnisse}

Die gezielte Überprüfung des Lernfortschritts der Teilnehmer gehört zu jeder Art von Unterricht. Dabei wird versucht, herauszufinden, ob ein tatsächlicher Lehrerfolg durch Informationszuwachs, Beherrschung von Fertigkeiten oder sachgemäßes Verhalten erreicht werden konnte. Was den AV-Sektor betrifft, so bietet sich hier vor allem das Gespräch im Anschluß an den Medieneinsatz an. Die Schüler beziehen Stellung zum behandelten Thema und zeigen durch die Verwendung sachbezogener Begriffe, sowie durch die Beurteilung der Situation, ob sie einen Lerngewinn erzielen konnten. Auch mit Hilfe eines Fragebogens, z. B. nach der Filmvorführung, kann der Lerneffekt kontrolliert werden. Einen besonderen Fall stellt der programmierte Unterricht dar, bei dem Zuwendung und Aufmerksamkeit durch eine fortlaufende Erfolgsbestätigung bewirkt wird. Z wischen der Situationserhebung am Beginn und der Rückmeldung am Ende des Lernvorgangs müßte für den Lehrer ein Wissenszuwachs, eine differenzierte Meinung bzw. eine Verhaltensänderung von seiten der Schüler feststellbar sein.

\section{Berücksichtigung der Lerndimensionen und Lernstufen}

Die rationale Vermittlung allein genügt auch in der religiösen Bildungsarbeit nicht. Gerade im Religionsunterricht ist es ja wichtig, daß neben den kognitiven Lernzielen auch den affektiven und pragmatischen eine bedeutsame Rolle zukommt. Klar ist, daß Prozesse im rationalen, emotionalen oder pragmatischen Bereich nicht genau voneinander geschieden werden können; vielmehr handelt es sich bei dieser Aufteilung eher um Akzentsetzungen, bei der jede Lerndimension von den anderen durchdrungen und mitbeeinflußt wird. Besonders bedeutsam scheint mir heute, daß der intellektualistische Unterricht abgelöst wird durch eine ganzheitliche Ansprache des Menschen, die auch den emotionalen Bereich und die unterbewußten Schichten erfaßt. Die Totalsprache der AVM berühren den Menschen nicht nur im kognitiven, sondern auch im emotionalen und voluntativen Bereich.

\section{Die sachgemäße Repräsentation von Unterrichtsinhalten}

Medien sind Mittler. Sie haben die Aufgabe, den Lernstoff zu repräsentieren. Die Vergegenständlichung von Unterrichtsinhalten ist notwendig, um bei den Teilnehmern eine Vorstellungsgrundlage über den anfallenden Lerngegenstand zu schaffen. Pestalozzi spricht in dem Zusammenhang von der „Anschauung als Fundament der Erkenntnis". Wegen seiner psychosomatischen Ganzheit ist der Mensch auch bei der geistigen Tätigkeit auf sinnenhafte Wahrnehmungen und Vorstellungen angewiesen. Die AVM entsprechen solchen Erwartungen, indem sie über die realitätsbezogene Konfrontation der Schüler mit dem Lehrgegenstand zu einem Erlebnis der Wirklichkeit führen. Die Vermittlung solcher Erfahrungsgrundlagen für die Rezipienten bedeutet aber keineswegs, daß nur die Begegnung und Auseinandersetzung mit einem Stück Wirklichkeit ermöglicht wird; es geht vielmehr immer auch um die Erschließung und Aktualisierung früherer, persönlicher Erfahrungen der Teilnehmer. Es versteht sich von selbst, daß in der religiösen Bildungsarbeit nur solche AVM zum Einsatz kommen, deren Inhalte einer kritischen Prüfung standhalten. Ein Tonbild über das Alte Testament muß beispielsweise sowohl der Archäologie wie auch der Exegese und Dogmatik gerecht werden. Gute biblische Bilder sind auch mehr als nur Anschauungsmittel oder Illustrationen, die sich in pseudohistorischen, geographischen oder kulturgeschichtlichen Details erschöpfen. Will ein Medienproduzent der 
Eigenart biblischer Texte gerecht werden, so wird er ihre kerygmatische Intention verstehen lernen und in der Lage sein, das im Text verborgene Kerygma zum Ausdruck zu bringen. Vom Medienproduzenten auf theologischem Gebiet wird eine Bildexegese gefordert, die über das Auge zum Sinngehalt der Botschaft Jesu führt.

Die Berücksichtigung des Adressatenkreises

Bei Auswahl und Einsatz von AVM müssen Alter, Geschlecht, Reifegrad und Umwelt unbedingt in Rechnung gestellt werden. Es gilt immer wieder zu fragen: Wird durch die Verwendung des Mediums der Erfahrungshorizont der Zielgruppe angesprochen? Welche Bedeutung hat das Medium für Lehrende und Lernende in ihrer Lebenswelt? Wer solche Vorfragen außer acht läßt und ohne vorherige Information einen Kurzfilm einsetzt, wird häufig Schiffbruch erleiden. Es zeigen sich jeweils andere Wirkungen, ob eine Schallplatte mit einer Ansprache von Karl Rahner über den Tod vor Teilnehmern an einem Glaubensseminar oder vor Grundschulkindern eingesetzt wird.

\section{Eigenart und Eigenwirkung der AVM}

Der Medienforscher McLuhan behauptet, daß jedes Zeitalter durch eine bestimmte Form der Kommunikation geprägt wird. Der Buchdruck, das Telefon, das Fernsehen haben das Gleichgewicht $\mathrm{zwischen}$ den menschlichen Sinnen jeweils verändert und einem von ihnen einen neuen Akzent gegeben. Die geistigen Rezeptionsstrukturen der Menschen und ihre Art, Wirklichkeit zu erfahren, und zu erleben, hat dadurch eine jeweils andere Prägung erhalten. Akustische, optische oder multisensorische Informationsträger führen $\mathrm{zu}$ jeweils andersgearteten Erlebnissen. Audiovisuelle Medien haben ihre Eigen-Art und bringen dadurch spezifische Eigen-Wirkungen hervor.

Eine Aufschlüsselung der Medien nach verschiedenen Arten, ergibt folgendes Bild: Unisensorische (einsinnige) Medien sprechen jeweils nur ein menschliches Sinnesorgan an. Dazu zählen sowohl akustische (auditive) Medien - wie z. B. Funk, Schallplatte, Tonband, Minikassette - die sich an den Gehörsinn wenden; wie auch optische (visuelle) Medien - wie z. B. Fotos, Bildkarten, Dias, Plakate, bedruckte Folien, Stummfilme - die sich an den Gesichtssinn wenden. Multisensorische Medien beanspruchen dagegen mehrere Sinnesorgane; hierzu zählen Tonfilme, das Fernsehen, die Bildplatte, der Videorecorder.

Die Erfahrung zeigt, daß die Totalsprache des Films tatsächlich bis in die unterbewußten Schichten unseres Daseins reicht und den Menschen nicht nur im kognitiven, sondern auch im emotionalen und voluntativen Bereich berührt.

\section{Das Verhältnis zwischen Medien und Methoden}

Durch den Einsatz von Medien soll mit Hilfe bestimmter Verfahren eine Begegnung zwischen dem Schüler und dem Unterrichtsgegenstand hergestellt werden. Bevor Medien ausgewählt werden, muß eine Überprüfung daraufhin erfolgen, mit welchen Methoden sie vorzugsweise eingesetzt werden können. Medien dürfen nämlich dem angewandten Verfahren nicht widersprechen. Durch die Entscheidung für eine unsachgemäße Methode wird der Bildungseffekt der Medien verhindert. Um es $\mathrm{kraß} \mathrm{zu}$ sagen: Mit einer Schallplatte althebräischer Melodien läßt sich keine Textanalyse durchführen.

Die Aussageübermittlung durch AVM begünstigt eine Umorientierung vom frontalen zum kooperativen Unterricht, vom autokratischen zum sozialintegrativen Unter- 
richtsstil. AVM begünstigen das Gruppengespräch und die Gruppenarbeit. Spannende Hörspiele, zeitnahe Fernsehreportagen, Problemfilme usw. haben eine starke gesprächsauslösende Wirkung. Kindergärtnerinnen und Lehrer werden am Montag mit Äußerungen über Fernsehsendungen des Wochenendes eingedeckt. Aber auch in der Welt der Erwachsenen liefern Rundfunkkommentare und Fernsehspiele sehr häufig Gesprächsstoff. Für die Unterrichtsplanung sollte deshalb in Rechnung gestellt werden, daß der gezielte Einsatz von AVM das Gespräch nicht verhindert, sondern provoziert.

Bei einer häufigen Variation von Methoden und Medien spricht man von KontextModellen. Dabei werden zum Teil neue Verfahren, wie z. B. Telekolleg, mit herkömmlichen Unterrichtsmitteln, wie z. B. dem Lehrbuch, kombiniert. Der Vorteil so strukturierter Unterrichtsprogramme liegt u. a. darin, daß der einseitige Gebrauch bestimmter Medien verhindert wird. Die lernhemmenden Wirkungen einzelner Methoden und Medien werden durch den Wechsel in bestimmten Grenzen ausgeglichen.

\section{Disponibilität und Organisationsaufwand}

Für den täglichen Gebrauch von AVM in Unterricht und Bildung spielt das Verhältnis von Organisationsaufwand und Effektivität eine große Rolle. Zwar wird die positive Wirkung von AVM im Lernprozeß heute nicht mehr in Frage gestellt; dagegen bereitet die Beschaffung von Medien und ihr reibungsloser Einsatz immer noch erhebliche Schwierigkeiten. Schon die Besorgung der nötigen Geräte kann lästig werden. Oft mangelt es auch an technischen Kenntnissen für den Umgang mit Apparaten. Nicht zu ibersehen sind schließlich die finanziellen Aufwendungen. Barrieren dieser Art haben bisher die verstärkte Verwendung von AVM in der Bildungsarbeit gebremst.

\section{Die Bedeutung des Gesprächs}

Das Gespräch ist auch heute noch die höchste Form menschlicher Kommunikation, die Urform allen Lehrens und Lernens und das bedeutendste Mittel für die Erziehung. Wenn auch immer wieder von einem visuellen Zeitalter gesprochen wird, so darf man darunter keinesfalls verstehen, daß das Wort seine Bedeutung verliert und daß die AVM den Lehrer oder den Referenten in der Bildungsarbeit einfach ersetzen könnten. Im Gegenteil, das Bild kann ja nur selten ohne eine Erklärung existieren. Dazu besitzt es meist zu viele Interpretationsmöglichkeiten. Rhythmen und Klänge sind ebenfalls vieldeutig. AVM bedürfen der Deutung und Verarbeitung durch das Gespräch. Der gezielte Einsatz audiovisueller Medien wertet das Gespräch nicht ab, sondern auf. Medium heißt ja "Mittler“, ist also nicht Selbstzwedk. Darum dürfen AVM auch niemals zu "Selbstläufern" werden. Es zeigt sich immer wieder, daß Medien dann am effektivsten wirken, wenn über das Dargebotene intensive Gespräche geführt werden. So wird in einer Zeit voll technischer Perfektionen und mechanisierter Vermittlungen das Gespräch in Erziehung und Bildung nicht vernachlässigt, sondern erhält einen neuen Stellenwert.

\section{Anmerkungen:}

1. Vgl. R. Tilmann: Sozialer und religiöser Wandel. Düsseldorf 1972, S. 59.

2. F. Zödhbauer: Der deutsche Kurzfilm. München 1969/1970.

3. Vgl. P. Heimann: Didaktik als Theorie und Lehre, in: Die Deutsche Schule 54; W. Schulz: Unterricht - Analyse und Planung. Hannover ${ }^{4} 1969$. 


\section{S U M M A R Y}

The importance of audio-visual media for the learning process and for formation activities of the Churches has become more and more clear in the last years. The author studies the special functions of these media in the religious learning process and develops two interesting lists of criteria: the first enumerates criteria which allow the use of AV-media in religious instructions; the second shows when, and under what conditions the use of these media theologically cannot be responsibly supported. The obvious advantage for the use of AV media, according to the author lies on the fact that they foster relation to reality, and also on the possibilities for religious language and expression, as well as sensitizing for basic experiences of human life. The article concludes with an extensive presentation and analysis of criteria which should guide selection and use of AV media in religious formation in a relatively new field of religious education.

\section{RESUME}

L'importance des moyens audio-visuels pour les études religieuses et les manifestations de formation ecclésiastique s'est confirmée au cours des dernières années. Anton Täubl éclaircit les fonctions particulières de ces mass media pour les processus des études religieuses et dresse deux listes de critères très intéressantes. La première liste présente tous les critères qui font des moyens audio-visuels des moyens appropriés ł l'enseignement religieux; une deuxième liste énumère quand et à quelles conditions on ne peut répondre de l'usage de ces moyens en théologie. L'auteur voit les avantages décisifs pour la mise en application des moyens audiovisuels dans le fait qu'ils peuvent favoriser le rapport avec la réalité et les possibilités d'expression orale et écrite, de même qu'ils peuvent sensibiliser les expériences fondamentales de l'homme. Un exposé et une analyse complète des critères qui doivent diriger le choix et la mise en place des moyens audio-visuels dans le travail de formation ecclésiastique concluent cet exposé en en faisant un domaine relativement nouveau de l'enseignement religieux.

\section{R E S UM E N}

Durante los últimos años se puso de manifiesto, cada vez con mayor evidencia, la importancia de los medios de comunicaciòn social audiovisuales en los procesos didácticos religiosos y en cursillos de formación organizados por la Iglesia. Anton Täubl analiza las funciones esenciales de estos medios en los procesos de formación religiosa y establece dos listas de criterios muy interesantes: La primera incluye todos los criterios que hacen adecuados los medios audiovisuales a la enseñanza religiosa; en la segunda lista enumera cuándo y bajo que circunstancias no puede justificarse desde el punto de vista teológico el empleo de tales medios. El autor considera que las ventajas de peso para el uso de medios audiovisuales residen en el hecho de que exigen la reflexión sobre la realidad y las posibilidades del lenguaje y de la expresión religiosa y pueden sensibilizar las experiencias básicas humanas. Concluye esta exposición sobre un campo relativamente nuevo del publicismo eclesiástico una presentación exhaustiva y el análisis de criterios que deben dirigir la elección y empleo de medios audiovisuales en el trabajo formativo religioso. 